\title{
Le transfert des signaux mitogéniques : une affaire de particules
}

Les récepteurs de facteur de croissance possédant une activité intrinsèque de tyrosine kinase s'associent au niveau de leur domaine cytoplasmique à d'autres protéines participant au transfert des signaux mitogéniques. La constitution de ces "particules" chargées de recevoir, de traiter et de transmettre la stimulation mitogénique dépend de l'occupation du récepteur par son ligand et de l'autophosphorylation qui en résulte. La nature des complexes polyprotéiques est différente selon les récepteurs et, peut-être, pour un même récepteur, selon les cellules. Cela permet de mieux expliquer la spécificité fonctionnelle de ces récepteurs et la modulation de leur activité selon les tissus.

\section{Odile Filhol}

\section{Claude Cochet}

\section{RÉFÉRENCES}

1. Meisenhelder J, Suh PG, Rhee SG, Hunter T. Phospholipase C- $\gamma$ is a substrate for the PDGF and EGF receptor proteintyrosine kinases in vivo and in vitro. Cell $1989 ; 57: 1108-22$

2. Wahl MI, Olashaw NE, Nishibe S, Rhee SG, Pledger WJ, Carpenter G. Platelet derived growth factor induces rapid and sustained tyrosine phosphorylation of phospholipase C- $\gamma$ in quiescent BALB/c 3T3 cells. Mol Cell Biol 1989 ; 9 : 2934-43.

\section{ADRESSE}

O. Filhol : boursière post-doctorale ligue nationale contre le cancer. $\mathrm{C}$. Cochet : directeur de recherche à l'Inserm. Inserm U. 244, Brce/lbio/dbms, centre d'études nucléaires, 85X, 38041 Grenoble Cedex, France.

\section{TIRÉS A PART}

e déclenchement de la division cellulaire met en jeu des protéine kinases spécifiques des sérines et des thréonines, mais, aussi certaines protéine kinases spécifiques des tyrosines (oncogènes de la famille src, récepteurs membranaires pour l'EGF, le PDGF, le FGF, l'insuline, le CSF, l'IGF1, etc. (pour une revue, consulter $\mathrm{m} / \mathrm{s}$, suppl. au $n^{\circ} 7$, vol. 3, p. 23).

Au cours du mois de mars 1990 s'est tenu au, Salk Institute (San Diego, USA), un congrès célébrant dix ans de recherche sur les protéine kinases spécifiques des résidus tyrosine. Dix années d'efforts qui ont permis de mettre en lumière le rôle crucial joué par les récepteurs membranaires des facteurs de croissance et les tyrosine protéine kinases intracellulaires, mais également dix années de frustration pour ce qui est de l'identification des substrats cellulaires de ces tyrosine protéine kinases.

Cependant, une étape importante a été récemment réalisée dans l'élucidation du mécanisme de transduction du signal mitogénique. Plusieurs protéines cibles, substrats du récepteur du PDGF et possédant des activités enzymatiques ont été identifiées : une isoforme $(\gamma)$ de la phospholipase $C[1,2]$, la sérine kinase cRaf [3], la MAP sérine kinase [4-7], la phosphatidyl inositol 3-kinase [8, 9] et la protéine $\operatorname{GAP}[10,11]$ qui règle l'activité GTPase du protooncogène Ras.

L'enjeu actuel est dès lors de déterminer quelle est l'importance de ces phosphorylations pour ce qui est de la réponse cellulaire. Ce problème complexe a été récemment abordé en étudiant les mécanismes biochimiques par lesquels les récepteurs pour les facteurs de croissance déclenchent l'activation de sérine kinases et la stimulation du métabolisme des polyphospho-inositides.

\section{Les récepteurs des facteurs de croissance participent à la formation de complexes}

Des résultats récents provenant de plusieurs laboratoires indiquent que les récepteurs tyrosine kinases s'associent et sont copurifiés avec certaines de leurs cibles primaires. Il est, en $\mathrm{m} / \mathrm{s} \mathrm{n}^{\circ} 10$, vol. 6 , décembre 90 
effet, possible de détecter une série de protéines peu abondantes qui sont coimmunoprécipitées avec les récepteurs stimulés par leur ligand, mais ces mêmes protéines sont absentes dans les immunoprécipités de récepteurs non stimulés. Certaines de ces protéines associées aux récepteurs du PDGF et de l'EGF ont été identifiées.

La phospholipase C- $\gamma$ (PLC- $\gamma$ ) s'associe au domaine cytoplasmique des récepteurs du PDGF [12] et de l'EGF [13], et il existe une corrélation entre cette association et la capacité qu'ont ces récepteurs à activer le cycle des poly-phosphoinositides. L'activité tyrosine kinase des récepteurs est nécessaire pour déclencher cette association avec la PLC- $\gamma$. De plus, ces récepteurs mutés au niveau de leurs sites d'autophosphorylation sont incapables de s'associer à la PLC- $\gamma$ [12]. La PLC- $\gamma$, comme plusieurs protéines cytoplasmiques qui participent à la transduction de signaux mitogéniques, possède deux régions homologues à celle initialement décrite dans la partie non catalytique de la $\mathrm{P} 60^{\text {c-src }}$ kinase, appelée domaine SH2 (SRC homology region 2) [14]. On suspecte que ce type de séquence possède un rôle régulateur permettant des interactions protéine- protéines. Dans le cas de la PLC- $\gamma$, une hypothèse séduisante consiste à envisager que les domaines $\mathrm{SH} 2$ de cette enzyme lui permettent d'interagir avec les régions des récepteurs porteuses de sites autophosphorylables. Dès lors, l'activation du récepteur et l'autophosphorylation qui en résulte représenteraient les facteurs déclenchants pour l'association entre les deux molécules (figure 1).

Plusieurs groupes ont montré qu'une activité phosphatidyl-inositol kinase (PI kinase) se retrouvait dans des immunoprécipités du récepteur de l'EGF [15] et du PDGF [16]. Cette enzyme phosphoryle la position D3 du PI-4-phosphate [9]. Elle forme des complexes avec les formes activées des récepteurs pour le PDGF [17], l'EGF [18], l'insuline [19], le CSF [20]. L'activation de cette enzyme permet la production de phosphatidyl-inositol 3-phosphate et de phosphatidyl-inositol 3,4-bisphosphate qui ne sont pas des substrats de la phospholipase C- $\gamma$.

Le rôle de cette phosphatidyl-inositol 3-kinase est encore mal connu. Toutefois, il existe une étroite corrélation entre son association au récepteur du PDGF et l'activité mitogénique de ce facteur de croissance [21]. De plus, l'association de cette enzyme avec l'antigène moyen $\mathrm{T}$ du polyome [16] et avec la p60 v-src [22] semble nécessaire au pouvoir transformant de ces oncogènes, ce qui indique que la phosphatidyl-inositol 3-kinase joue vraisemblablement un rôle important dans le contrôle du signal mitogénique.

Le produit du protooncogène ras, qui est une protéine de liaison du GTP, est impliqué dans le contrôle de la prolifération cellulaire. En effet, la micro-injection d'anticorps monoclonaux anti-Ras dans des fibroblastes bloque leur prolifération induite par le sérum, le PDGF et l'EGF [23]. Une protéine appelée GAP, qui stimule l'activité GTPase de la forme cellulaire normale de Ras, est phosphorylée sur des résidus tyrosine dans des fibroblastes stimulés par EGF ou PDGF [10, 11]. Des résultats récents montrent que la protéine GAP, qui possède également des domaines $\mathrm{SH} 2$, appartient à la liste des protéines qui forment un complexe avec le récepteur du PDGF. Cette association nécessite une activité tyrosine kinase fonctionnelle ainsi que la phosphorylation des deux résidus tyrosine correspondant aux sites d'autophosphorylation du récepteur

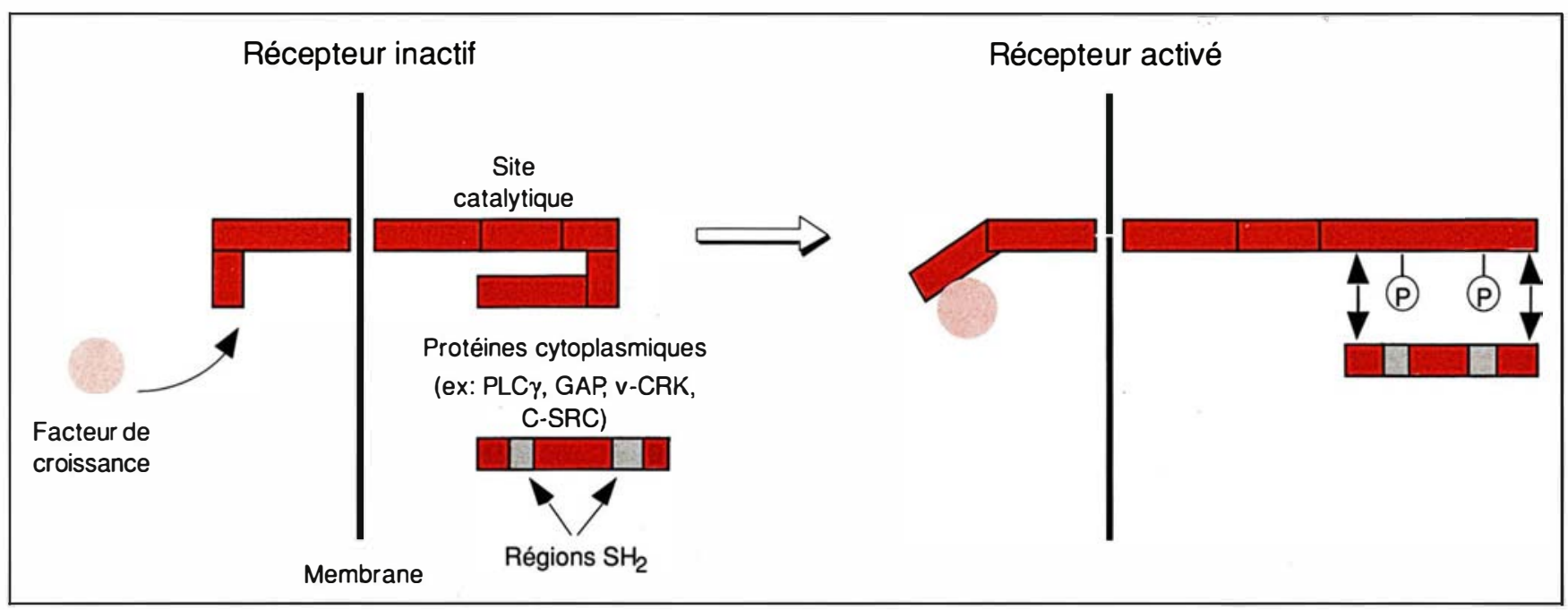

Figure 1. Un des mécanismes envisagés pour expliquer l'association entre des protéines cytoplasmiques et les récepteurs activés des facteurs de croissance. La liaison du facteur de croissance sur le domaine externe du récepteur membranaire induit un changement conformationnel du domaine cytoplasmique de la molécule qui permet I'activation de sa fonction tyrosine kinase. L'autophosphorylation du récepteur qui en résulte permet I'association du domaine cytoplasmique du récepteur avec certaines protéines qui possèdent des séquences de type SH2 (SRC homology region 2). 


\section{RÉFÉRENCES}

3. Morrison DK, Kaplan DR, Escobedo JA, Rapp UR, Roberts TM, Williams LT. Direct activation of the serine/threonine kinase activity of Raf-1 through tyrosine phosphorylation by the PDGF receptor. Cell 1989 ; 58 : 649-57. 4. Rossomando AJ, Payne DM, Weber MJ, Sturgill TW. Evidence that pp42, a major tyrosine kinase target protein is a mitogen-activated serine/threonine protein kinase. Proc Natl Acad Sci USA $1989 ; 86: 6940-3$.

5. Anderson NG, Maller JC, Tonks NK, Sturgill TW. Requirement for integration of signals from two distinct phosphorylation pathways for activation of MAP-kinase. Nature 1990 ; 343 : 651-3.

6. Kahn A. MAP-kinase, un intégrateur possible des différentes voies de stimulation de la division cellulaire. médecine/sciences $1990 ; 6: 392-5$.

7. Boulton TG, Yancopoulos GD, Gregory JS, et al. An insulin-stimulated protein kinase similar to yeast kinases involved in cell cycle control. Science $1990 ; 249$ : 64-7. 8. Coughlin SR, Escobedo JA, Williams LT. Role of phosphatidyl-inositol kinase in PDGF signal transduction. Science $1989 ; 243$ : 1191-4.

9. Auger KR, Serunian LA, Soltoff SP, Libby P, Cantley L. PDGF-dependent tyrosine phosphorylation stimulates production of novel polyphosphoinositides in intact cells. Cell 1989; 57 : 167-75.

10. Molloy CJ, Bottaro DP, Fleming TP, Marshall MS, Gibbs JB, Aaronson SA. PDGF induction of tyrosine phosphorylation of GTPase activating protein. Nature 1989 ; $342: 711-4$.

11. Ellis C, Moran M, McCornick F, Pawson T. Phosphorylation of GAP and GAPassociated proteins by transforming and mitogenic tyrosine kinases. Nature 1990 ; 343 : 377-81.

12. Morrison DK, Kaplan DR, Rhee SG, Williams LT. Platelet-derived growth factor (PDGF)-dependent association of phospholipase C- $\gamma$ with the PDGF receptor signaling complex. Mol Cell Biol 1990; 10 : 2359-66.

13. Margolis RL, Bellot F, Honegger AM, Uhlrich A, Schlessinger J, Zilberstein A. Tyrosine kinase activity is essentiel for the association of phospholipase C- $\gamma$ with the epidermal growth factor receptor. Mol Cell Biol 1990 ; 10 : 435-41.

14. Koch CA, Moran M, Sadowski I, Panson $T$. The commun src homology region 2 domain of cytoplasmic signaling proteins is a positive effector of $\mathrm{v}$-fps tyrosine kinase function. Mol Cell Biol 1989; 9 : 4131-40. 15. Thompson DM, Cochet C, Chambaz EM, Gill GN. Separation and characterization of a phosphatidyl-inositol kinase activity that co-purifies with the EGF receptor. J Biol Chem 1985 ; 260 : 8824-30. 16. Kaplan DR, Whitman M, Schaffhausen B, et al. Common elements in growth factor stimulation and oncogenic transformation : $85 \mathrm{Kd}$ phosphoprotein and phosphatidyl-inositol kinase activity. Cell $1987 ; 50$ : 1021-9.

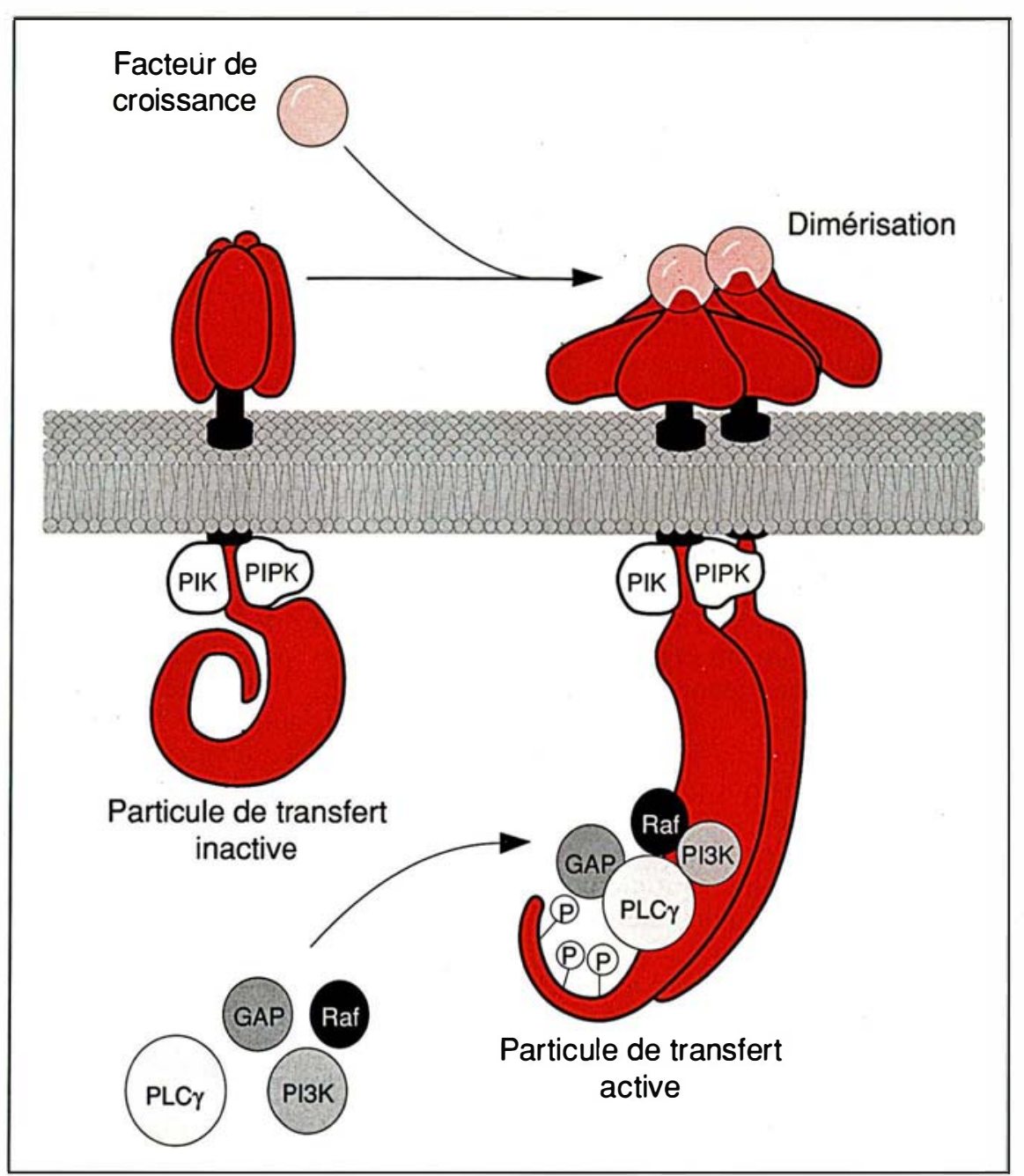

Figure 2. Un modèle de particule de transfert de signal mitogénique. Certaines enzymes telles que la phosphatidyl-inositol kinase (PIK), la phosphatidylinositol-4 phosphate kinase (PIPK) sont liées au domaine cytoplasmique du récepteur formant une particule de transfert inactive. L'activation du récepteur par le ligand entraîne son association avec plusieurs protéines cytoplasmiques qui sont des substrats du récepteur telles que la phospholipase $C-\gamma$ $(P L C-\gamma)$, la phosphatidyl-inositol 3-kinase (PI3K), la protéine activatrice du Ras (GAP) et le produit du proto-oncogène c-Raf. Cette association avec le récepteur activé permet l'assemblage d'une particule de transfert active, nécessaire au déclenchement des événements moléculaires qui conduisent à la réponse cellulaire. (D'après Ullrich et Schlessinger [29].)

[24]. Ces résultats suggèrent que la protéine GAP doit représenter un intégrateur commun pour les voies de transfert de signaux mitogéniques utilisées par Ras et celles mises en jeu par les récepteurs tyrosine kinases. Une hypothèse serait que la liaison de GAP à un récepteur membranaire diminuerait sa capacité à activer l'hydrolyse du GTP lié à la protéine Ras. Les conséquences de cette association seraient alors une stabilisation temporaire de la forme active de Ras (Ras-GTP) qui participe au transfert du signal mitogénique.

Parmi les protéines phosphorylées sur des résidus tyrosine dans des cellules stimulées par PDGF, la sérine/thréonine kinase $\operatorname{Raf}-1$ participe à la transduction du signal mitogénique déclenchée par ce facteur de croissance [25].

Des résultats récents démontrent que la protéine kinase Raf-1 est directe 
ment phosphorylée sur des résidus tyrosine par le récepteur du PDGF et s'associe physiquement avec lui pour former un complexe [3]. Làencore, l'autophosphorylation du récepteur du PDGF est nécessaire à la formation du complexe.

Cette association entre les deux protéines et la phosphorylation de la protéine $\mathrm{Raf}-1$ sur des résidus tyrosine stimule son activité sérine/thréonine kinase. Il est également concevable que cette association serve à positionner Raf-1 au niveau de la membrane plasmique où les substrats de cette kinase peuvent se trouver localisés.

\section{Les particules de transfert de signaux mitogéniques}

Différentes approches indiquent que les cibles des récepteurs tyrosine kinases sont des composants de structures supramoléculaires que l'on peut caractériser comme des "particules de transfert de signaux" (figure 2). Bien que nouvelle dans le domaine des facteurs de croissance, cette notion d'assemblage moléculaire a été abondamment décrite pour d'autres voies métaboliques. Pour ne citer qu'un exemple, le métabolisme des hexoses et trioses phosphates est réglé par l'assemblage et le désassemblage dynamique de complexes enzymatiques [26]. Les complexes observés sont souvent transitoires et rendus possibles grâce à la concentration élevée de ces enzymes dans le cytoplasme, ce qui favorise la formation de complexes protéine-protéines. Le rôle de l'assemblage de ces complexes est, dans ce cas, de créer une "tunnélisation " qui permet une ségrégation et un transfert efficaces des métabolites [26].

De par leur taille volumineuse, les domaines cytoplasmiques de récepteurs de facteurs de croissance offrent une surface suffisante pour des interactions multiples avec d'autres protéines. De plus, la liaison du facteur de croissance déclenche pour la plupart de ces récepteurs un phénomène de dimérisation susceptible de participer à la formation des complexes observés [27, 28]. Cependant, le fait que les protéines retrouvées associées aux récepteurs des facteurs de croissance soient peu abondantes laisse supposer que l'assemblage de ces $m / s n^{\circ} 10$, vol. 6 , décembre 90

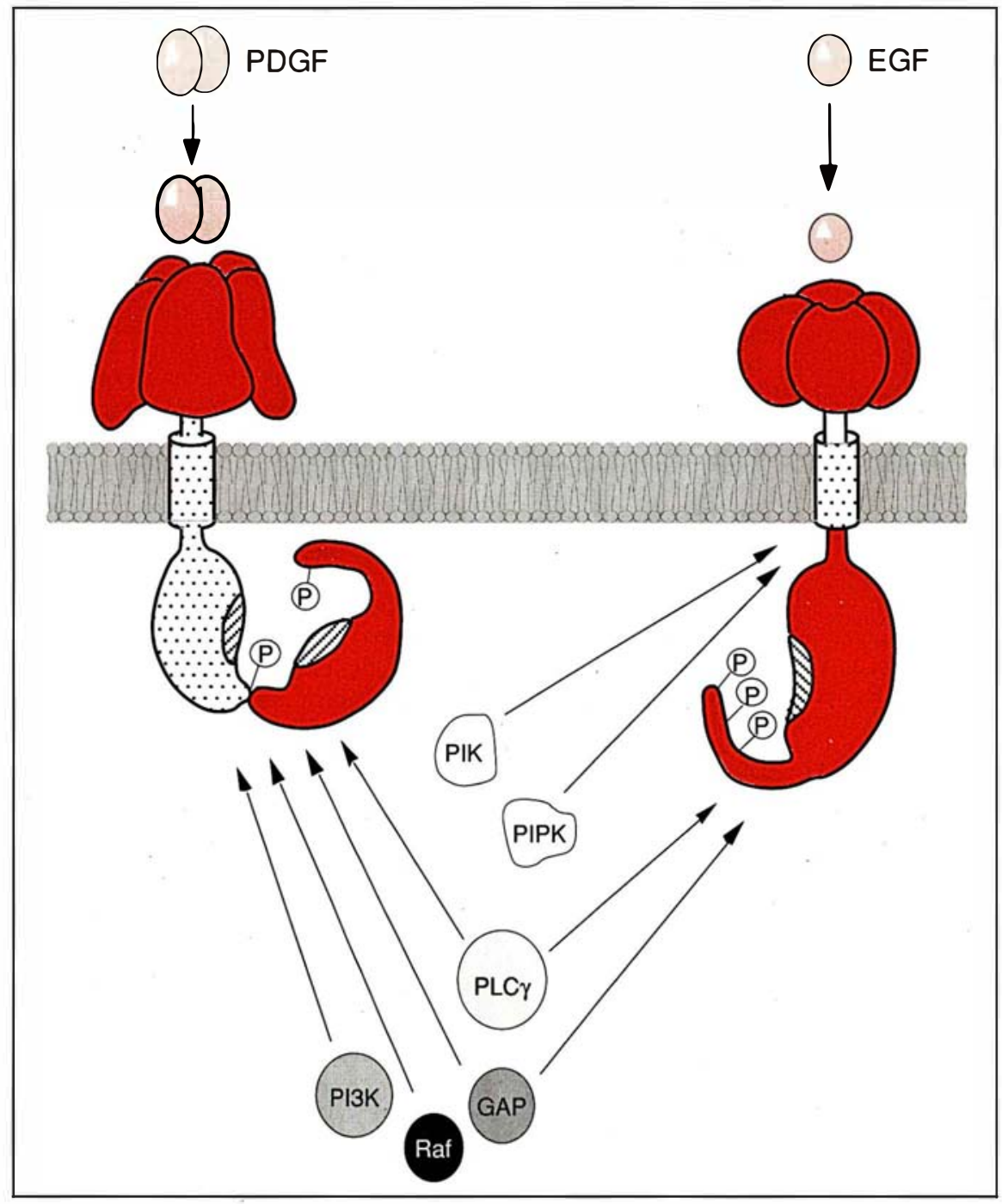

Figure 3. Spécificité dans l'assemblage des particules de transfert. L'activation des récepteurs du PDGF et de l'EGF conduit à un assemblage différentiel des complexes moléculaires qui forment la particule de transfert et à des effets biologiques communs ou spécifiques de chaque facteur de croissance [30].

particules met en jeu des mécanismes biochimiques particuliers. Il est possible, par exemple, que des formes latentes d'enzymes soient associées aux récepteurs non stimulés. C'est le cas pour la phosphatidyl-inositol kinase et la phosphatidyl-inositol 4-phosphate kinase. Nous avons pu montrer que ces enzymes sont associées à la forme non activée du récepteur de l'EGF. Elles sont également retrouvées associées à divers récepteurs mutants dépourvus d'activité tyrosine kinase. Cela signifie que des protéines peuvent être associées de façon constitutive au domaine cytoplasmique des récepteurs et forment alors des "particules de transfert inactives".

$\mathrm{Au}$ contraire, l'activation de ces récepteurs déclenche leur interaction spécifique avec d'autres protéines et, par là-même, modifie la composition de la particule de transfert. L'assemblage dynamique de la particule de transfert active peut faire appel à deux types de mécanismes:

(1) le facteur de croissance se lie à son récepteur et l'autophosphorylation qui en résulte induit un changement conformationnel de son domaine cytoplasmique qui démas- 


\section{RÉFÉRENCES}

17. Kazlauska A, Cooper JA. Autophosphorylation of the PDGF receptors in the kinase insert region regulates interactions with cell proteins. Cell $1989 ; 58: 1122-33$ 18. Bjorge JD, Chan TO, Antczack KM, Kung HJ, Fijita DJ. Activated type I phosphatidyl-inositol kinase is associated with the epidermal growth factor receptor following EGF stimulation. Proc Natl Acad Sci USA 1990, in press.

19. Ruderman NB, Kapeller R, White MF, Cantley LC. Activation of phosphatidylinositol 3-kinase by insulin. Proc Natl Acad Sci USA 1990; 87 : 1411-5.

20. Varticovski L, Drucker B, Morrison D, Cantly L, Roberts $\mathrm{T}$. The colony stimulating factor-1 receptor associates with and activates phosphatidyl-inositol 3-kinase. Nature 1990 ; 342 : 699-702.

21. Williams LT. Signal transduction by the platelet-derived growth factor receptor. Science 1989 ; 243 : 1564-70

22. Fukui Y, Hanafusa H. Phosphatidylinositol kinase activity associates with vira p60 src protein. Mol Cell Biol 1989; 9 : 1651-8.

23. Mulcahy LS, Smith MR, Stacey DW Requirement for ras proto-oncogene function during serum stimulated growth of NIH 3T3 cells. Nature 1985 ; 313 : 241-3. 24. Kazlauskas A, Ellis C, Pawson T, Cooper JA. Binding of GAP to activated PDGF receptor. Science 1990 ; $247: 1578-81$.

25. Morrison DK, Kaplan DK, Rapp U, Roberts TM. Signal transduction from membrane to cytoplasm : growth factors and membrane-bound oncogene products increase Raf-1 phosphorylation and associated protein kinase activity. Proc Natl Acad Sci USA 1988 ; 85 : 8855-9.

26. Ovadi J. Old pathway-new concept : control of glycolysis by metabolitemodulated dynamic enzyme associations. TIBS $1988 ; 13$ : 486-90.

27. Schlessinger J. Signal transduction by allosteric receptor oligomerization. Trends Biochem Sci 1988 ; 13: 443-7.

28. Cochet C, Kashles O, Chambaz EM, Borrello I, King CR, Schlessinger J. Demonstration of epidermal growth factorinduced receptor dimerization in living cells using chemical covalent cross-linking agent. $J$ Biol Chem 1988 ; 263 : 3290-5.

29. Ullrich A, Schlessinger J. Signal transduction by receptors with tyrosine kinase activity. Cell 1990; 61 : 203-13.

30. Kaplan DR, Morrison DK, Wong G, McCornick F, Williams LT. $\mathrm{PDGF}_{\beta}$ receptor stimulates tyrosine phosphorylation of GAP and association of GAP with a signaling complex. Cell $1990 ; 61$ : 125-33.

31. Filhol O, Cochet C, Chambaz EM. La pathologie des insectes au service du génie génétique. médecine/sciences $1990 ; 6$ :

que des sites permettant la fixation de plusieurs protéines;

(2) les charges négatives apportées par l'autophosphorylation du récepteur constituent un signal de reconnaissance pour des motifs présents dans la séquence de diverses protéines impliquées dans le transfert du signal mitogénique. C'est le cas des séquences de type $\mathrm{SH} 2$ discutées précédemment (figure 1).

De plus, certains de ces récepteurs possèdent la propriété de passer par un état oligomérique en réponse à leur ligand spécifique [27, 28], ce qui constitue un moyen supplémentaire de moduler ces interactions hétérologues.

En conclusion, la notion nouvelle qui se dégage est que les récepteurs des facteurs de croissance "travaillent" en très étroite association avec des protéines impliquées dans la génération de messagers secondaires, avec des produits de proto-oncogènes ou des facteurs qui contrôlent l'activité de proto-oncogènes. Ces associations conduisent à l'assemblage de particules de transfert de signaux mitogéniques dont le rôle est probablement de localiser et de contrôler l'activité enzymatique des protéines associées dans le complexe. D'ores et déjà, on peut noter que la spécificité des récepteurs se retrouve également au niveau de l'assemblage de la particule de transfert, ce qui signifie que la particule formée en réponse à $\mathrm{PDGF}$ sera différente de celle que déclenchera l'activation du récepteur de l'EGF (figure 3, p. 983). Par conséquent, les effets biologiques communs et spécifiques de chaque facteur de croissance peuvent s'expliquer par les protéines spécifiques qui s'associent à leurs récepteurs activés [30]. Certaines de ces protéines participent directement au transfert et à la propagation du signal mitogénique, mais il est également vraisemblable que certaines enzymes du complexe contrôlent le destin intracellulaire de la particule dont l'existence à la surface membranaire n'est que transitoire. La production en masse des protéines associées aux récepteurs des facteurs de croissance dans le système d'expression du baculovirus [31] permet dès à présent l'étude in vitro du rôle et de la régulation de l'assemblage de ces particules fonctionnelles

\section{Summary}

Growth factor receptor-mediated assembly of signal transfer particles

Despite the best efforts of a large number of laboratories using a variety of experimental approaches, we still do not know exactly how growth factors mediate their pleiotropic actions. However, a recent meeting on tyrosine phosphorylation held in San Diego California, brought a glimmer of light into the darkness which will certainly lead to greater insight into signal transduction by growth factor receptors. Among the proteins identificd thus far as receptor tyrosine kinase targets, some of them, which are involved in activation of diverse signaling pathways, like phospholipase $\mathrm{C} \gamma$, the serine threoninc kinases c-Raf and MAP II kinase, the phosphatidylinositol 3-kinase, the ras activating protein GAP, associate with the cytoplasmic domain of the receptors to form " signal transfert particles ". Data indicate that the extent of tyrosine phosphorylation of the tyrosine kinase receptors correlates with their ability to associate with some cellular proteins which have in common homologous $\mathrm{SH} 2 / \mathrm{SH} 3$ sequences found initially in the non catalytic domain of the p60 ${ }^{\text {c-src }}$ kinase. It is therefore suspected that these types of sequences have a regulatory role in the assembly of the active signal transfer particles. Binding of growth factors of their specific receptors leads to the activation of their tyrosine kinase activity together with a rapid oligomerisation of the receptor molecules. However, this receptor activation promotes the interaction of its cytoplasmic domain with different cellular proteins and the composition of the resulting signal transfer particle might reflect the distinct and common signaling pathways triggered by different growth factors. The mechanisms and functional consequences of these dynamic processes of assembly are discussed. 